\title{
A GENERAL MODEL OF MATING BEHAVIOUR WITH NATURAL SELECTION AND FEMALE PREFERENCE
}

\author{
P. O'DONALD \\ Department of Genetics, University of Cambridge, Downing Street, \\ Cambridge CB2 $3 E H$, England
}

Received 26.ix.77

\begin{abstract}
SuMMARY
A model is analysed in which female mating preferences are expressed partially, depending on the frequencies of the males. A female, who has been stimulated sufficiently to mate with a male of the phenotype she prefers, will only mate with another male after a certain number of extra encounters with the others. After $n$ of these extra encounters, females with preferences mate randomly with any male. The probability that $n$ encounters occur depends on the frequencies of the different male phenotypes. If $n=1$, the mating advantage gained by the preferred males rises slightly as their frequency increases: either natural selection against the preferred phenotypes outweighs their mating advantage and they are eliminated, or they spread through the population to fixation; stable polymorphisms cannot become established. If $n>1$, the mating advantage declines with increasing frequency: stable polymorphisms can exist over a range of values of the parameters of mating preference and natural selection. As $n$ increases, the mating advantage becomes more strongly frequencydependent and a wider range of values of parameters gives rise to stable polymorphisms. When $n>5$, the results are very close to those of models in which mating preferences are always expressed. The rates of selection and equilibrium frequencies are the same for preferred characters determined either by dominant or by recessive alleles. In the models the preferred character is either sex-limited and expressed only in males or expressed in both sexes. If it is expressed in both sexes, the effect of natural selection is doubled, and twice as many females must have the mating preference before the preferred character may start to spread through the population.
\end{abstract}

\section{INTRODUCTION}

MANY of the published models of sexual selection and assortative mating have been derived on the assumption that a certain proportion of individuals mate preferentially with those who have particular genotypes or phenotypes (O'Donald, 1960, 1962, 1967, 1973, 1974; Karlin, 1969; Karlin and Scudo, 1969; Scudo and Karlin, 1969). Experiments have usually shown that only the females exercise any preference for their mates (Ehrman, 1966, 1967, 1968, 1969, 1972; Spiess, 1968; Spiess and Spiess, 1969). The mating preferences are complete if preferential mating always takes place whenever a choice can be made between the preferred individuals and the others. According to a more general model (O'Donald, 1976; Wedd, 1977), the expression of preference may only be partial: females are ready to mate after they have encountered a certain number of courting males; a female expresses a preference for males with particular characteristics if she only mates with other males after extra encounters with them.

If some females have different thresholds at which they respond to different male phenotypes (O'Donald, 1977a, 1977b), they will mate prefer- 
entially by responding more readily to some males than to others. Each successive encounter with a courting male will increase a female's general level of stimulation until she is ready to mate. If she then encounters a male with the characteristics she prefers, she mates with him. But if she has a higher threshold against other males, extra encounters will be needed before she is sufficiently stimulated to mate with one of these other males. If certain genotypes or phenotypes are the object of the mating preferences, the parameters of the model are the proportions $\alpha, \beta, \ldots$ of females with preferences for each genotype or phenotype, and the number $n$ of the extra encounters necessary to stimulate these females to mate at random among all the males. In the most realistic model, a mating preference for a particular character of sexual display is counterbalanced by natural selection. Thus the red throat of the male stickleback in the breeding season acts as a sexual stimulus to the females, but red-throated males suffer more predation than non-red-throated males (Semler, 1971; Moodie, 1972). Many characters for sexual display probably reduce an individual's chances of survival. In this paper, I analyse genetical models of a deleterious character that increases the chances of mating according to the general mechanism of mate selection I have proposed. The character may be sex-limited to males or expressed in both sexes but only the females may exercise a mating preference in favour of individuals possessing the preferred character.

\section{Desaription OF THE MODELS}

The mating system is assumed to be polygynous: a male who has been chosen as a mate is then replaced in the pool of males from which the females in turn make their choice. Suppose $A$ and $B$ are the two phenotypes of males occurring at frequencies $x$ and $y$ in the population. A proportion $\alpha$ of the females prefer the $A$ males as mates and $\gamma$ prefer the $B$ males. A female, who has been sufficiently stimulated to mate with the males she prefers, will not, however, mate with other males until she has had another $n$ successive encounters with them. Thus $n$ is the number of the extra encounters necessary to raise her level of stimulation to the point at which she will accept any male and mate at random. The two phenotypes of males mate with the following probabilities.

Frequencies of matings with $A$ males: $\alpha\left(1-y^{n+1}\right)+\gamma x^{n+1}+x(1-\alpha-\gamma)$

Frequencies of matings with $B$ males: $\alpha y^{n+1}+\gamma\left(1-x^{n+1}\right)+y(1-\alpha-\gamma)$.

In the models, $A$ is a dominant character, genotypically $A A$ or $A a$, and $B$ is a recessive, genotypically $a a$. Thus if the frequencies of the genotypes $A A$, $A a$ and $a a$ are $u, v$ and $w$ when mate selection takes place, the probabilities that females select males of each of these genotypes are then as follows:

$\begin{array}{ccc}\text { Genotypes } & \text { Frequencies } & \text { Probabilities of mating of males } \\ A A & u & \alpha\left(\frac{u}{u+v}\right)\left(1-w^{n+1}\right)+\gamma\left(\frac{u}{u+v}\right)(1-w)^{n+1}+\sigma u \\ A a & v & \alpha\left(\frac{v}{u+v}\right)\left(1-w^{n+1}\right)+\gamma\left(\frac{v}{u+v}\right)(1-w)^{n+1}+\sigma v \\ a a & w & \alpha w^{n+1}+\gamma\left[1-(1-w)^{n+1}\right]+\sigma w\end{array}$


where $\sigma=1-\alpha-\gamma$. O'Donald (1978) obtained the expressions for the change in gene frequency in this model for the cases when $n=1$ and $n=2$. As $n \rightarrow \infty$, the model reduces to that with complete preferences (O'Donald, 1977a, 1977b). If $p$ is the frequency of the allele $A$, then it can be shown that for a change in one generation

$$
\begin{gathered}
\text { when } n=1, \quad \Delta p=\frac{1}{2} p w(\alpha-\gamma) \\
\text { when } n=2, \quad \Delta p=\frac{1}{2} p w[\alpha-2 \gamma+w(\alpha+\gamma)] \\
\text { as } n \rightarrow \infty, \quad \Delta p=\frac{1}{2} p[-\gamma+w(\alpha+\gamma)] /(1-w) .
\end{gathered}
$$

When $n=1$, there is clearly no polymorphic equilibrium: if $\alpha>\gamma, p_{n} \rightarrow 1$; if $\alpha<\gamma, p_{n} \rightarrow 0$. This follows because the selection is not strongly frequencydependent: either the $A$ phenotype or the $B$ phenotype has the overall advantage which is retained at all frequencies. Consider the preference for the $A$ phenotype alone. When $n=1$, and the phenotype is rare, its selective advantage is $\alpha /(1+\alpha)$. When it has become common, its selective advantage has increased slightly to $\alpha$. Thus no polymorphism can be maintained by mating preferences alone when $n=1$, since there is slight positive frequencydependence.

When $n=2$, however, the equilibrium frequency

$$
p_{*}=1-\sqrt{(2 \gamma-\alpha) /(\alpha+\gamma)}
$$

is stable if $\frac{1}{2} \gamma<\alpha<2 \gamma$ giving a range of polymorphic equilibria. The preference for $A$ when $n=2$ produces a selective advantage of $2 \alpha /(1+2 \alpha)$ when $A$ is rare. This declines to $\alpha$ when $A$ is common. The frequencydependence is negative so long as $\alpha<\frac{1}{2}$, giving stability to the equilibrium frequency. As $n$ increases, so does the negative frequency-dependence, leading to the stable equilibrium

$$
p_{*}=1-\sqrt{\gamma /(\alpha+\gamma)}
$$

for values of $n>10$.

Mating preferences for alternative phenotypes can thus produce stable polymorphisms. In some computer models in which alleles at one locus determine preferences for genotypes at another locus, preferences for two different characters can evolve (O'Donald, 1977b): in some other models the preferences evolve only for one of the characters. Unless there has been introgression of populations with preferences for different characters, it seems more likely that one specific character, like the red-throat of the stickleback would be the object of the preference. Since characters for sexual stimulation and display must often reduce the viability of their possessors, natural selection must also be incorporated into the model. For generality of the model, natural selection must act differently against each of the preferred phenotypes. But in the simplest realistic model, a character which is the object of female preference is also selectively disadvantageous, either in males alone if sex-limited, or in both sexes if not. If a recessive character is preferred, females select males of each genotype with the following probabilities: 
Frequencies

Genotypes after selection

Probabilities of mating of males

$\begin{array}{ll}A A & \frac{u}{1-t w} \\ A a & \frac{v}{1-t w} \\ a a & \frac{w(1-t)}{1-t w}\end{array}$

$$
\begin{aligned}
& \gamma\left(\frac{u}{u+v}\right)\left[1-\frac{w(1-t)}{1-t w}\right]^{n+1}+\frac{\sigma u}{1-t w} \\
& \gamma\left(\frac{v}{u+v}\right)\left[1-\frac{w(1-t)}{1-t w}\right]^{n+1}+\frac{\sigma v}{1-t w} \\
& \gamma\left\{1-\left[1-\frac{w(1-t)}{1-t w}\right]^{n+1}\right\}+\frac{\sigma w(1-t)}{1-t w}
\end{aligned}
$$

where $\sigma=1-\gamma$ and $t$ is the selective disadvantage of the preferred genotype aa. If a dominant is preferred, then the corresponding probabilities are as follows:

$$
\begin{array}{ccc}
\text { Genotypes } & \begin{array}{c}
\text { Frequencies } \\
\text { after selection }
\end{array} & \text { Probabilities of mating of males } \\
A A & \frac{u(1-s)}{1-s(1-w)} & \alpha\left(\frac{u}{u+v}\right)\left\{1-\left[\frac{w}{1-s(1-w)}\right]^{n+1}\right\}+\frac{\sigma u(1-s)}{1-s(1-w)} \\
A a & \frac{v(1-s)}{1-s(1-w)} & \alpha\left(\frac{v}{u+v}\right)\left\{1-\left[\frac{w}{1-s(1-w)}\right]^{n+1}\right\}+\frac{\sigma v(1-s)}{1-s(1-w)} \\
a a & \frac{w}{1-s(1-w)} & \propto\left[\frac{w}{1-s(1-w)}\right]^{n+1}+\frac{\sigma w}{1-s(1-w)}
\end{array}
$$

where $s$ is the selective disadvantage of the genotypes $A A$ and aa. Assuming there is no assortment of the preference among the genotypes and hence no tendency to assortative mating, the females mate either with genotypic frequencies $u, v$ and $w$ if the character is sex-limited and natural selection acts only upon males or with the genotypic frequencies after selection if natural selection acts on both sexes. The frequencies of the matings and hence of the genotypes in the next generation can thus be found for particular values of $n$. Analytical solutions for equilibrium conditions seem to be obtainable only if $n=1$ or $n=2$ or if $n \rightarrow \infty$.

\section{Females mate at RANDom AFter one ENGounter$$
(n=1)
$$

In these models, females, who have been sufficiently stimulated to mate preferentially with the males of their choice, will then mate at random with any male after only one additional encounter with another male: the extra encounter raises the females' level of stimulation-or lowers their threshold-so that they are ready to accept any male. Thus if some females differ in the threshold of their receptivity to the male phenotypes, then this difference must be less than the stimulus provided by an encounter with one courting male: having been stimulated to the level of the lower threshold, the extra encounter raises the stimulation above both thresholds so that mating can take place with any male. 
(i) Preference for a recessive character

The following probabilities of the matings of males are obtained:

$\begin{array}{cl}\text { Genotypes } & \text { Probability of mating of males } \\ A A & u[1-\gamma w-t w(1-\gamma)] /(1-t w)^{2} \\ A a & v[1-\gamma w-t w(1-\gamma)] /(1-t w)^{2} \\ a a & w(1-t)[1-\gamma w-t w(1-\gamma)] /(1-t w)^{2} \\ & +\gamma w(1-t) /(1-t w)\end{array}$

If the preferred character is sex-limited, this gives a change of gene frequency from one generation to the next of

$$
\Delta p=\frac{1}{2} p w\left[t-\gamma(1-t)-t^{2} w\right] /(1-t w)^{2}
$$

with an unstable equilibrium at

$$
w_{*}=[t-\gamma(1-t)] / t^{2} .
$$

Thus the preferred character increases in frequency if $\Delta p<0$ and hence if

$$
\gamma>t /(1-t) \text {. }
$$

If this condition holds, the preferred character always increases to fixation in the population. No stable polymorphism can become established.

If the preferred character is expressed in both sexes, then

$$
\begin{aligned}
& \Delta p=\frac{1}{2} p w\left[2 t-\gamma(1-t)-2 t^{2} w\right] /(1-t w)^{2} \\
& w_{*}=\left[t-\frac{1}{2} \gamma(1-t)\right] / t^{2} \quad \text { (unstable) }
\end{aligned}
$$

and the character increases in frequency in the population if $\gamma>2 t /(1-t)$. As expected, natural selection has twice the effect if it acts on both sexes. The preference must then be twice as strong if it is to overcome the deleterious effects of the preferred character.

\section{(ii) Preference for a dominant character}

When a dominant character is preferred, the following probabilities of the matings of males are then obtained:

$$
\begin{array}{cl}
\text { Genotypes } & \multicolumn{1}{c}{\text { Probability of mating of males }} \\
A A & u(1-s)[1+\alpha w-s(1-w)] /[1-s(1-w)]^{2} \\
A a & v(1-s)[1+\alpha w-s(1-w)] /[1-s(1-w)]^{2} \\
a a & w[1+\alpha w-s(1-w)] /[1-s(1-w)]^{2} \\
& -\alpha w /[1-s(1-w)] .
\end{array}
$$

For a sex-limited character

$$
\Delta p=-\frac{1}{2} p w\left[s-\alpha(1-s)-s^{2}(1-w)\right] /[1-s(1-w)]^{2} .
$$

Thus

$$
1-w_{*}=[s-\alpha(1-s)] / s^{2} .
$$


This equilibrium is unstable, like that for a recessive. It also represents the same frequency of the preferred phenotype as that of the recessive given the same preference for the character and the same natural selection against it. The preferred character always increases in frequency if $\alpha>s(1-s)$. The equations for both dominant and recessive can be expressed in the forms

and

$$
\Delta p=-\frac{1}{2} t^{2} p w\left(w-w_{*}\right) /[1-t w]^{2}
$$

$$
\Delta p=-\frac{1}{2} s^{2} p w\left(w-w_{*}\right) /[1-s(1-w)]^{2} .
$$

Thus, given $s=t$ and $\alpha=\gamma$, the rate of selection is similar for both. In all cases the Hardy-Weinberg frequencies

$$
\begin{aligned}
u_{*} & =p_{*}^{2} \\
v_{*} & =2 p_{*} q_{*} \\
w_{*} & =q_{*}^{2}
\end{aligned}
$$

are maintained at equilibrium.

Similar results are obtained if the dominant character is expressed in both sexes. Then

$$
\Delta p=-\frac{1}{2} p w\left[2 s-\alpha(1-s)-2 s^{2}(1-w)\right] /[1-s(1-w)]^{2} .
$$

As when the preferred character is recessive, the preference must be twice as strong to overcome natural selection acting on both sexes. The preferred character always increases in frequency if

$$
\alpha>2 s /(1-s) \text {. }
$$

The rate of selection is about the same as when the character is recessive and expressed in both sexes.

\section{Females mate at random after two encounters

$$
(n=2)
$$

In these models, the difference in the thresholds of females' receptivity to the two phenotypes is greater than the stimulus provided by one encounter with a courting male, but less than the stimulus provided by two encounters. Thus a female who is ready to mate with a male of the type she prefers will still require two more encounters with other males before mating with one of them.

\section{(i) Preference for a recessive character}

The expressions for the probabilities of the matings of males are complicated, but can be reduced to the following:

Genotypes

$A A$

$A a$

$a a$
Probability of mating of males

$$
\begin{gathered}
u\left[1-2 w(\gamma+t-\gamma t)+w^{2}\left(\gamma+t^{2}-\gamma t^{2}\right)\right] /(1-t w)^{3} \\
v\left[1-2 w(\gamma+t-\gamma t)+w^{2}\left(\gamma+t^{2}-\gamma t^{2}\right)\right] /(1-t w)^{3} \\
w\left[1-2 w(\gamma+t-\gamma t)+w^{2}\left(\gamma+t^{2}-\gamma t^{2}\right)\right] /(1-t w)^{3} \\
+w(1-t)(2-w-t w) /(1-t w)^{3}-t w /(1-t w)
\end{gathered}
$$


These values give the change in gene frequency for a sex-limited character

$$
\Delta p=\frac{1}{2} p w\left[-2 \gamma+t+2 \gamma t+w\left(\gamma-2 t^{2}-\gamma t^{2}\right)+t^{3} w^{2}\right] /(1-t w)^{3} .
$$

Since $w$ is the frequency of the preferred phenotypes, its equilibrium is given by the solution of the quadratic

$$
t^{3} w^{2}+w\left(\gamma-2 t^{2}-\gamma t^{2}\right)-2 \gamma+t+2 \gamma t=0 .
$$

Since the first term is usually negligible, a good approximation is given by

$$
w_{*} \simeq(2 \gamma-t-2 \gamma t) /\left(\gamma-2 t^{2}-\gamma t^{2}\right) \text {. }
$$

Using this approximation

At equilibrium

$$
\Delta p=\frac{1}{2} p w\left(w-w_{*}\right)\left(\gamma-2 t^{2}-\gamma t^{2}\right) .
$$

and since

$$
w_{*}=q_{*}^{2}
$$

$$
\frac{d w}{d p}=-2 q \quad \text { near equilibrium }
$$

after differentiation

$$
\frac{d \Delta p}{d p}=-\left(\gamma-2 t^{2}-\gamma t^{2}\right) p_{*} q_{*} w_{*} .
$$

The equilibrium will therefore be stable if

$$
\gamma-2 t^{2}-\gamma t^{2}>0
$$

or

$$
\gamma>2 t^{2} /\left(1-t^{2}\right)
$$
found:

If $t=0.05$, the following polymorphic equilibrium values of $w_{*}$ are

\begin{tabular}{clc}
$\begin{array}{c}\text { Values } \\
\text { of } \gamma\end{array}$ & \multicolumn{1}{c}{ Exact } & $\begin{array}{c}\text { By approximate } \\
\text { equation }\end{array}$ \\
0.025 & 0 & 0 \\
0.030 & 0.2804 & 0.2808 \\
0.035 & 0.5503 & 0.5516 \\
0.040 & 0.7430 & 0.7450 \\
0.045 & 0.8875 & 0.8900 \\
0.050 & 1.0 & 1.0
\end{tabular}

Thus the approximate values given by the equation

$$
w_{*} \simeq(2 \gamma-t-2 \gamma t) /\left(\gamma-2 t^{2}-\gamma t^{2}\right)
$$

are reasonably close to the exact values and very close for smaller values of $t$. A polymorphism will be established if $\gamma$ lies approximately within the range of values.

$$
\frac{1}{2} t /(1-t)<\gamma<t(1-2 t) /(1-t)^{2} .
$$

The condition $\gamma>\frac{1}{2} t /(1-t)$ for initial increase of the preferred character implies the stability of the polymorphic equilibrium for the values $t<\frac{1}{3}$. 
When the preferred character is expressed in both sexes, then we find after much algebra that

$$
\Delta p=p w\left[-\gamma+t+\gamma t+w\left(\frac{1}{2} \gamma-2 t^{2}-\frac{1}{2} \gamma t^{2}\right)+t^{3} w^{2}\right] /(1-t w)^{3} .
$$

This equation can be obtained by substituting $\frac{1}{2} \gamma$ for $\gamma$ in the expression for $\Delta p$ for a sex-limited character and multiplying the whole expression by $2:$ as in previous examples, when the character is not sex-limited, the preference for it must be twice as large to produce the same effect. It then increases in frequency if $\gamma>t /(1-t)$, and given the stability condition

$$
\gamma>4 t^{2} /\left(1-t^{2}\right)
$$

reaches the approximate equilibrium frequency

$$
w_{*}=(\gamma-t-\gamma t) /\left(\frac{1}{2} \gamma-2 t^{2}-\frac{1}{2} \gamma t^{2}\right) \text {. }
$$

The condition for initial increase implies the stability condition for all values of $t<\frac{1}{3}$.

\section{(ii) Preference for a dominant character}

After reduction, we obtain the following expressions for the probabilities of matings of males:

Genotypes

Probability of mating of males

$A A$

$$
u(1-s)\left[(1-s)^{2}+w(1-s)(\alpha+2 s)+w^{2}\left(\alpha+\alpha s+s^{2}\right)\right] /[1-s(1-w)]^{3}
$$

$A a$

aa

$$
\begin{aligned}
& v(1-s)\left[(1-s)^{2}+w(1-s)(\alpha+2 s)+w^{2}\left(\alpha+\alpha s+s^{2}\right)\right] /[1-s(1-w)]^{3} \\
& w\left[(1-s)^{2}+w(1-s)(\alpha+2 s)+w^{2}\left(\alpha+\alpha s+s^{2}\right)\right] /[1-s(1-w)]^{3} \\
& -\alpha w\left[(1-s)^{2}+w(1-s)(1+2 s)+s w^{2}(1+s)\right] /[1-s(1-w)]^{3}
\end{aligned}
$$

These give the change in gene frequency for a sex-limited character

$\Delta p=-\frac{1}{2} p w\left[-2 \alpha+s+2 \alpha s+(1-w)\left(\alpha-2 s^{2}-\alpha s^{2}\right)+s^{3}(1-w)^{2}\right] /[1-s(1-w)]^{3}$ giving the same stable equilibrium frequency of the preferred phenotype under the same conditions, as that for a sex-limited recessive.

Similarly, when the character is expressed in both sexes,

$$
\Delta p=-p w\left[-\alpha+s+\alpha s+(1-w)\left(\frac{1}{2} \alpha-2 s^{2}-\frac{1}{2} \alpha s^{2}\right)+s^{3}(1-w)^{2}\right] /[1-s(1-w)]^{3}
$$

giving the same equilibrium and conditions as for a recessive.

\section{Females mate at random after several encounters}

As the number of extra encounters required for females to mate at random among the males is increased, so the advantage gained by being rare also increases: the sexual selection becomes more strongly frequencydependent and polymorphic equilibria are always stable. As $n \rightarrow \infty$ the females with preferences always mate preferentially. In the terms of the threshold model of mating responses, the difference in the thresholds between the preferred males and the rest is then many times the stimulus provided by a single encounter with a courting male. At the limit we obtain the following equilibrium frequencies: 
for a recessive sex-limited character

$$
w_{*}=\gamma / t
$$

for a recessive character expressed in both sexes

$$
w_{*}=\gamma / 2 t
$$

for a dominant sex-limited character

$$
1-w_{*}=\alpha / s
$$

for a dominant character expressed in both sexes

$$
1-w_{*}=\alpha / 2 s \text {. }
$$

As in previous sections, these are frequencies after mating and prior to the action of natural selection on the zygotes. Analytical details of these and other models of sexual selection have already been published (O'Donald, 1973, 1977b). The level of frequency-dependence found by Ehrman (1967), Spiess (1968) and Spiess and Spiess (1969) in their experiments on matings with Drosophila is in general agreement with that in the model with complete preference. However, in some of the data, a better fit to the general model with partial preferences can be obtained (O'Donald, 1978). Thus in Spiess and Spiess' data (1969) a model with $n=5$ seems to give the best fit.

In each of the particular models I have analysed $(n=1, n=2$ and $n \rightarrow \infty)$, the phenotypic equilibrium frequencies are the same for both dominant and recessive characters. We may conjecture that this is so for all values of $n$. The equilibria for particular preferences and selective values can easily be computed for any value of $n$. As expected the phenotypic equilibria were the same for both dominant and recessive characters preferred by the females. Table 1 shows the results of such computations for the case of a sex-limited character. It shows that there is rapid convergence on the equilibrium established with complete preferences $(n \rightarrow \infty)$ : for values of $n$ greater than five, the equilibrium is very close to that with complete preferences. The many models of assortative mating and sexual selection

\begin{tabular}{|c|c|c|c|c|c|c|c|c|c|}
\hline \multirow{2}{*}{$\begin{array}{c}\text { No. of } \\
\text { extra } \\
\text { encounters } \\
n\end{array}$} & \multicolumn{9}{|c|}{ Mating preferences for a character with a selective disadvantage of $10 \%$} \\
\hline & 0.01 & $0 \cdot 02$ & $0 \cdot 03$ & 0.04 & $0 \cdot 05$ & $0 \cdot 06$ & $0 \cdot 07$ & $0 \cdot 08$ & $0 \cdot 09$ \\
\hline 1 & 0 & 0 & 0 & 0 & 0 & 0 & 0 & 0 & 0 \\
\hline 2 & 0 & 0 & 0 & 0 & 0 & 0.202 & 0.522 & 0.734 & 0.886 \\
\hline 3 & 0 & 0 & 0 & 0.094 & 0.342 & 0.524 & 0.669 & $0 \cdot 791$ & 0.899 \\
\hline 4 & 0 & 0 & 0.061 & 0.279 & 0.442 & 0.575 & 0.692 & 0.798 & 0.900 \\
\hline 5 & 0 & 0 & 0.174 & 0.341 & 0.474 & 0.591 & 0.697 & $0 \cdot 800$ & 0.900 \\
\hline 10 & 0 & $0 \cdot 158$ & $0 \cdot 286$ & 0.396 & 0.499 & 0.600 & 0.700 & $0 \cdot 800$ & 0.900 \\
\hline 20 & 0.076 & $0 \cdot 196$ & $0 \cdot 300$ & 0.400 & 0.500 & 0.600 & 0.700 & $0 \cdot 800$ & 0.900 \\
\hline$\infty$ & $0 \cdot 1$ & $0 \cdot 2$ & $0 \cdot 3$ & 0.4 & 0.5 & 0.6 & 0.7 & 0.8 & 0.9 \\
\hline
\end{tabular}

TABLE 1

Phenotypic equilibrium frequencies of a character sex-limited to males aud maintained by female preference and selective disadvantage

The polymorphic equilibrium values shown here are all stable, but are reached only slowly at the smaller values of $n=1$ and $n=2$. They are all globally stable.

$40 / 3-\mathrm{G}$ 
that have been based on the assumption of complete preferences should therefore give widely applicable results. A model in which $n$ varied among the females should also give results close to those of the model with complete preferences provided that only a small proportion of females had values of $n$ less than about five.

\section{Discussion and GONGlusions}

According to the general model for the partial expression of female preference, the degree of the frequency-dependence is determined by the number of the extra encounters that a female must have with courting males before she mates randomly rather than preferentially between them. If she has different thresholds of receptivity to the different phenotypes, then the greater the difference between the thresholds, the more courtship she will need before she will be receptive to any male. The difference in the thresholds and hence the number of extra encounters needed to bridge this difference then determines the outcome of selection. If only one extra encounter is required, a slight positive frequency-dependence is produced in the mating advantage gained by the males who possess the preferred character. Either the natural selection is sufficient to outweigh this advantage or it is not. If the mating advantage is outweighed, then the preferred character must be eliminated: if not, the preferred character spreads through the population to eventual fixation. A stable polymorphism can in no way be maintained.

For all larger values of $n$, however, the mating advantage is negatively frequency-dependent: it declines with increasing frequency of the preferred males. Natural selection, which may not be sufficient to outweigh the mating advantage at first, may do so later, however, when the preferred males have become common and their advantage has been reduced. This may then produce a stable polymorphism. The range of values that give rise to polymorphism becomes wider as $n$ increases and the mating advantage varies more closely with frequency. The rates of selection and equilibrium frequencies are the same for preferred characters determined either by a dominant or by a recessive allele.

In all the foregoing analysis and discussion of the models, it has been assumed that females encounter a succession of courting males and either mate at random or exercise a preference between the male phenotypes. But of course there are other ways by which sexual selection might operate. Suppose groups of males display to the females. A female with a preference will then be able to mate preferentially in any group that contains at least one of the males she prefers. If the number of males in the group is $n+1$ this leads to exactly the same general model that $\mathbf{I}$ have analysed in sections 2, 3 and 4 of this paper: if $x$ and $y$ are the frequencies of the $A$ and $B$ phenotypes preferred by $\alpha$ and $\gamma$ of the females, then the probability that a group of $n+1$ males contains at least one $A$ male is $1-y^{n+1}$ the probability that it contains all $A$ males is $x^{n+1}$ and so the average probability that an $A$ male finds a mate is $\alpha\left(1-y^{n+1}\right)+\gamma x^{n+1}+x(1-\alpha-\gamma)$ as given in section 2. This model still assumes that some females do have preferences which they exercise if the males they prefer are available for mating.

Theoretical arguments have been put forward to account for the evolution of mating preferences (Fisher, 1930; O’Donald, 1967, 1977b). Mating 
preferences have been shown to evolve very quickly: the preference increases the advantage of the preferred males; this increases the selective advantage of the preference, producing Fisher's " runaway process" of sexual selection. On this theoretical basis, mating preferences should often be found to favour characters of sexual recognition and display which may therefore have evolved and been enhanced as a result. But mating preferences are not the only plausible mechanism of sexual selection: direct competition between males to exclude rivals from mating increases the contribution successfu] males can make to the next generation; the more rivals excluded the greater is the contribution of the successful competitor. Threat and other forms of display may determine the outcome of the competition, as well as actual fighting. Generally, threatening behaviour towards other males is closely correlated with sexual display towards the females. If males vary phenotypically in the intensity of their displays, this will produce sexual selection. Suppose the sexually more active males produce $k$ times the stimulus of the less active males: an encounter with one of the more active males raises a female's level of stimulation by an amount $x$ so that females who are ready to mate with these males must have been stimulated to within $x$ of their threshold. The other males stimulate a female by an amount $x / k$. If as a result of previous stimulation, females are at a point anywhere within $x$ of their threshold, then they will mate if they encounter one of the more active males. A proportion $(x / k) / x=1 / k$ of the females will also be at a point within $x / k$ of the threshold and will therefore mate if they encounter one of the less active males. The remaining proportion $(k-1) / k$ of these females will only mate with one of the less active males after one or more extra encounters with them. We may put $(k-1) / k=\alpha$ for this is the proportion of females who act as if they had a mating preference for the more active males. If $k \leqq 2$, only one extra encounter will be needed before all the remaining females mate at random: this would be formally equivalent to the model when $n=1$. If $k>2$, some females would require more than one extra encounter and the model would then be a mixed one with $n=1$ for some females, $n=2$ for some others, and so on. The models with partial expression of mating preferences can thus be used to describe a model of male competition.

\section{REFERENCES}

EHRMAN, L. 1966. Mating success and genotype frequency in Drosophila. Anim. Behav., 14, 332-339.

EHRMAN, L. 1967. Further studies on genotype frequency and mating success in Drosophila. Am. Nat., 101, 415-424.

ehrman, L. 1968. Frequency-dependence of mating success in Drosophila pseudoobscura. Genet. Res., Camb., 11, 135-140.

EHRMAN, L. 1969. The sensory basis of mate selection in Drosophila. Evolution, 23, 59-64.

ehrman, L. 1972. Genetics and sexual selection. Sexual Selection and the Descent of Man 1871-1971, ed. B. Campbell, pp. 105-135. Aldine, Chicago.

FISHER, R. A. 1930. The Genetical Theory of Natural Selection. Clarendon Press, Oxford.

KARLIN, s. 1969. Equilibrium Behavior of Population Genetic Models with Non-random Mating. Gordon and Breach, New York.

KARLIN, s., AND scudo, F. M. 1969. Assortative mating based on phenotype. II. Two autosomal alleles without dominance. Genetics, 63, 499-510.

MoodiE, G. E. E. 1972. Predation, natural selection and adaptation in an unusual Threespine stickleback. Heredity, 28, 155-167.

$40 / 3-\mathrm{G}^{*}$ 
o'DONALD, P. 1960. Assortative mating in a population in which two alleles are segregating. Heredity, 15, 389-396.

o'donald, P. 1962. The theory of sexual selection. Heredity, 17, 541-552.

o'Donald, P. 1967. A general model of sexual and natural selection. Heredity, 22, 499-518.

o'DonALD, P. 1973. Models of sexual and natural selection in polygamous species. Heredity, $31,145-165$.

o'DonALD, P. 1974. Polymorphisms maintained by sexual selection in monogamous species of birds. Heredity, 32, 1-10.

o'Donald, P. 1976. Mating preferences and their genetic effects in models of sexual selection for colour phases of the Arctic Skua. Population Genetics and Ecology, ed. S. Karlin and E. Nevo, pp. 411-430. Academic Press, New York.

o'Donald, P. 1977a. Mating advantage of rare males in models of sexual selection. Nature, 267, 151-154.

o'Donald, P. 1977b. Theoretical aspects of sexual selection. F. Theor. Pop. Biol., 12, 298-334. o'DONALD, P. 1978. Theoretical aspects of sexual selection: a generalised model of mating behaviour. 7. Theor. Pop. Biol. (in press).

SGUDo, F. M., AND KARLIN, s. 1969. Assortative mating based on phenotype. I. Two alleles with dominance. Genetics, 63, 479-498.

SEMLER, D. E. 1971. Some aspects of adaptation in a polymorphism for breeding colours in the Threespine stickleback (Gasterosteus aculeatus). F. Zoo.. Soc., Lond., 165, 291-302.

SPIEss, Е. в. 1968. Low frequency advantage in mating of Drosophila pseudoobscura karyotypes. Am. Nat., 102, 363-379.

SPIESS, L. D., AND SPIESS, E. B. 1969. Minority advantage in interpopulational matings of Drosophila persimilis. Am. Nat., 103, 155-172.

wedd, N. s. 1977. Population Dynamics and Sexual Selection in the Arctic Skua. M.Sc. Thesis, University of Cambridge. 